\title{
Reproductive aging and elective fertility preservation
}

Rani Fritz ${ }^{1}$ and Sangita Jindal ${ }^{1,2^{*}}$ (D)

\begin{abstract}
Reproductive aging is a natural process that occurs in all women, eventually leading to reproductive senescence and menopause. Over the past half century there has been a trend towards delayed motherhood. Postponing reproduction can increase the chance of a woman remaining involuntarily childless as well as an increase in pregnancy complications in those that do achieve pregnancy at advanced maternal age. Despite the well-documented decrease in fecundity that occurs as a woman ages, reproductive aged women frequently overestimate the age at which a significant decline in fertility occurs and overestimate the success of assisted reproductive technologies (ART) to circumvent infertility. Oocyte cryopreservation enables women to achieve genetically related offspring in the event that they desire to postpone their childbearing to an age after which a significant decline in fertility occurs or in circumstances in which their reproductive potential is compromised due to medical pathology. Available success rates and safety data following oocyte cryopreservation have been reassuring and is not considered experimental according to the American Society for Reproductive Medicine and the European Society for Human Reproduction and Embryology. This review article will focus on an evidence-based discussion relating to reproductive aging and oocyte cryopreservation.
\end{abstract}

Keywords: Reproductive aging- oocyte cryopreservation- reproduction

\section{Background}

Over the past several decades spanning from 1970 to 2014, the average age of first time mothers in the U.S. has increased $18.6 \%$ from 21.4-26.3 y/o [1, 2]. Alongside this trend, from 1970 to 2012 in the U.S. the percentage of women aged 35-44 y/o having children for the first time has increased more than five-fold from 2.5 to 13.3 per 1000 women [3]. Similar trends are also found in other parts of the world including Canada and Europe with one of the highest mean age of first time mothers seen in Italy at $30.6 \mathrm{y} / \mathrm{o}[4,5]$. These trends are likely related to an increase in women entering the workforce, obtaining advanced educational degrees, and greater use of contraceptive methods [6]. The declining birthrate is a concern for many developed countries. As birthrates in many industrialized countries fall below the replacement fertility rate of 2.1 live births per women, there is concern for future economic and social consequences including a decreased workforce to sustain the economy

\footnotetext{
* Correspondence: SJindal@montefiore.org

${ }^{1}$ Department of Obstetrics, Gynecology \& Women's Health, Albert Einstein College of Medicine/Montefiore Medical Center, Minneapolis, USA

${ }^{2}$ Montefiore's Institute for Reproductive Medicine and Health, New York, USA
}

and care for the elderly population $[7,8]$. It has therefore been advocated in Europe that a more liberal incorporation of assisted reproductive technologies (ART) into society can assist in achieving fertility rates towards replacement levels [8].

Women are born with a finite number of oocytes reaching their peak at 20 weeks gestation with 6-7 million and decreasing to 1-2 million at birth, 300,000500,000 at puberty, 25,000 by $37 \mathrm{y} / \mathrm{o}$, and approximately 1000 at menopause $[9,10]$. Alongside a quantitative decline in oocyte number, there is also a qualitative decline primarily attributed to an increase in aneuploidy amongst aging oocytes. A study evaluating ploidy status of over 1300 metaphase II oocytes revealed that aneuploidy rates remain relatively stable from 20 to $34 \mathrm{y} / \mathrm{o}$ ranging between $5.2-10 \%$, increasing to $12.5-28.1 \%$ at ages $35 \mathrm{y} / \mathrm{o}$ to $40 \mathrm{y} / \mathrm{o}, 50 \%$ at ages $42-43 \mathrm{y} / \mathrm{o}$, and $100 \%$ at $45 \mathrm{y} / \mathrm{o}$ [11]. A similar trend is seen in embryo aneuploidy rates, as a study evaluating over 15,000 consecutive embryo trophectoderm biopsies with comprehensive chromosomal screening revealed a dramatic increase in embryo aneuploidy rates at ages 35-37 y/o, approaching $90 \%$ at ages $43-45$ y/o [12]. The increase in oocyte and 
embryo aneuploidy in relation to age is likely related to a disturbance in meiotic competence due to abnormal meiotic spindle formation [13, 14].

Increase aneuploidy rates with ovarian aging results in decreased fecundity, with most epidemiological studies revealing a significant decline between 35 and $37 \mathrm{y} / \mathrm{o}$ [15]. The Hutterites are one of the most frequently referenced epidemiological cohorts. They immigrated form Switzerland and settled in the Dakotas and Montana in the 1870's and detailed records were kept relating to their childbearing [16]. They did not practice contraception and were a relatively fertile population with an average childbirth rate of 9.6 children per woman and a low an infertility rate of $2.4 \%$. In this cohort, a steep decline in fecundity was seen at 35-37y/o, with 1 in 10 woman stopping to reproduce by $35 \mathrm{y} / \mathrm{o}, 1$ in 3 by $40 \mathrm{y} / \mathrm{o}$ and 7 in 8 by 45 y/o [16]. This trend revealing a significant decrease in fecundity at $35-37 \mathrm{y} / \mathrm{o}$ is consistent with multiple historical epidemiological studies [17]. Recent cohort studies reveal similar trends. A time to pregnancy cohort study evaluating the fecundability of 960 women aged 30-44 y/o trying to conceive 3 months or less revealed stable pregnancy rates of $82-88 \%$ after 12 menstrual cycles in women aged 30-35 y/o, decreasing to $71-76 \%$ between 36 and $39 \mathrm{y} / \mathrm{o}$, and to $48-54 \%$ between $40-44 y / 0$ [18]. Interestingly, history of no prior pregnancy adversely affected fecundability rates. For example, in patients aged 38-39 y/o with a history of a prior pregnancy, $81 \%$ had a pregnancy after 12 cycles compared to $35 \%$ without a prior pregnancy, with similar trends seen in patients $\geq 40 \mathrm{y} / \mathrm{o}$ [18]. A major limitation of this study is that they did not report on live birth rates, as patients were only followed to a positive pregnancy test. With the known increase in miscarriage rates in older patient populations, live birth rates would have likely been significantly lower in the older patient populations. Other recent cohort studies reveal a similar trend with decreasing fecundability after 35 y/o [19, 20]. Limitations of the Hutterite study and other epidemiological studies are that they do not account for the possibility of decreasing sexual activity amongst aging women, however, a study evaluating intrauterine insemination (IUI) pregnancy rates using donor sperm amongst women with male partners suffering from azoospermia revealed a significant decrease in success rates after $35 \mathrm{y} / \mathrm{o}$ [21]. After 12 cycles, women $26-30 \mathrm{y} / \mathrm{o}$ had a success rate of $74.1 \%$, whereas, women over $35 \mathrm{y} / \mathrm{o}$ had a success rate $53.6 \%$. IUI success rates were defined as a positive pregnancy test and, as above, given the increase in miscarriage rates seen with advanced maternal age, if followed to live birth, the difference between both age groups would have likely widened. Another study evaluating pregnancy rates in 751 donor sperm cycles from couples with male infertility revealed a similar significant decline in pregnancy rates after 35 y/o [22].
Data from in vitro fertilization (IVF) cycles in the United States reveal a similar decrease in fecundity with advancing age. There is a persistent misconception that assisted reproductive technology can reverse the "aged biological clock" [23], however, IVF success rates decrease significantly as women enter their 5 th decade of life. U.S data from the Centers for Disease Control in 2015 reveal live birth rates per cycle of IVF of $33.1 \%$ in woman under 35 years old, decreasing to $8.3 \%$ at $41-42$ $\mathrm{y} / \mathrm{o}, 3.2 \%$ at $43-44 \mathrm{y} / \mathrm{o}$ and $0.8 \%>44 \mathrm{y} / \mathrm{o}$ [24]. Alongside these trends, there is an increase in the use of donor oocytes with advancing maternal age. Donor oocyte use is $10 \%$ or less $\leq 40 \mathrm{y} / \mathrm{o}$, increasing to $18 \%$ at $41-42 \mathrm{y} / \mathrm{o}$, $34 \%$ at $43-44 \mathrm{y} / \mathrm{o}$ and $71 \%$ over $44 \mathrm{y} / \mathrm{o}$ [24].

Involuntary childlessness is a major consequence of delayed motherhood. Psychologically and socially, involuntary childlessness is associated with higher rates of low self-esteem, depression, partner separation, and even higher rates of mortality [25]. There are also consequences of pregnancy at advanced maternal age to both the mother and newborn. Women $\geq 35 \mathrm{y} / \mathrm{o}$ are at increase risk for preeclampsia, gestational diabetes, placenta previa, placental abruption, operative deliveries including cesarean section, and even maternal deaths [26-29]. Women of advanced maternal age, particularly $\geq 40 \mathrm{y} / \mathrm{o}$ are also at increased risks of adverse neonatal outcomes including preterm birth, low birth weight, very low birth weight, neonatal intensive care unit admissions, fetal death, congenital anomalies, and increased risks of chromosomal abnormalities [26, 27, 29-32]. Spontaneous abortion, or miscarriage, also significantly increase with advance maternal age. Rates of clinical miscarriages are generally approximately $15 \%$, however, increase to $20 \%$ by $35 \mathrm{y} / \mathrm{o}$, and $50 \%$ by age $42 \mathrm{y} / \mathrm{o}$ [33]. Miscarriage rates in infertile populations following IVF are similar to those seen in the non-infertile population, as miscarriage rates following IVF are below $15 \%$ in women $<35 \mathrm{y} / \mathrm{o}$, and rise to $30 \%$ at $40 \mathrm{y} / \mathrm{o}$ and $65 \%$ in women older than $44 \mathrm{y} / \mathrm{o}$ [24]. Although generally not life threatening, miscarriages may result in severe psychological burden. A survey evaluating patients with miscarriages revealed that $37 \%$ felt that they had lost a child, $47 \%$ felt guilt, $41 \%$ felt alone, and $28 \%$ felt ashamed [34]. This psychological burden may be more devastating in women of advanced maternal age struggling with infertility. Additionally, miscarriages following IVF in women of advanced maternal age may have adverse effects on the success of subsequent treatments as a delay of 6 months can cause a significant decrease in the quality and quantity of ovarian reserve.

Although it is well documented that advanced maternal age decreases a woman's chance to conceive, studies from multiple countries consistently reveal that reproductive aged women have suboptimal knowledge relating 
to reproductive aging, particularly underestimating age-related fertility decline, and over-estimating the success of IVF [35-42]. One of the largest surveys evaluating over 10,000 women and men from 79 countries revealed an average score of $56 \%$ on a 13 item fertility knowledge questionnaire [43]. Knowledge relating to reproductive aging and IVF success outcomes have also been shown to be suboptimal amongst obstetrics and gynecology $(\mathrm{OB} / \mathrm{GYN})$ US residents. A study evaluating $238 \mathrm{OB} / \mathrm{GYN}$ residents revealed that although approximately $73 \%$ believed that a conversation relating to fertility decline with aging should be part of a well-woman exam, $47.5 \%$ underestimated the age at which a marked decline in fertility occurs, and $78.4 \%$ over-estimated the success of IVF [44].

\section{Premature accelerated loss of fertility, risk factors, ovarian reserve markers}

Although multiple markers for ovarian reserve have been evaluated, age is likely the most predictive marker for ovarian reserve and the ability to conceive. The average age of menopause in Western countries is approximately 51 years of age [45]. Approximately $10 \%$ will experience menopause by $45 \mathrm{y} / \mathrm{o}$, and $1 \%$ will experience primary ovarian insufficiency (POI) defined as menopause by $40 \mathrm{y} / \mathrm{o}[46,47]$. It is estimated that there is an accelerated decline in fertility 13 years prior to the onset of menopause, and thus approximately $10 \%$ of woman will experience an accelerated decline in fertility at around $32 \mathrm{y} / \mathrm{o}$ [46]. Ideally these patients would be easily identified so that they can be counseled on reproductive aging and the availability of elective fertility preservation, however, currently there are no optimal tests to identify these cohort of women. Obtaining an adequate medical history may identify women at risk for POI or an accelerated loss of fertility. Women diagnosed with cancer that will undergo chemotherapy or pelvic radiation, or patients that have undergone these treatments are at risk and warrant counseling with a reproductive specialist relating to elective fertility preservation. Other patients that may be at risk for accelerated fertility decline include severe endometriosis, ovarian surgery, strong smoking history, family history of early menopause, Turner Syndrome or Turner mosaic, and Fragile X pre-mutation [48-53].

Day 3 follicle stimulating hormone (FSH) alongside estradiol $\left(\mathrm{E}_{2}\right)$, antral follicle count (AFC), and anti-mullerian hormone $(\mathrm{AMH})$ are all frequently utilized markers of ovarian reserve; however they cannot predict when a significant decline in fertility will begin to occur and are more predictive of ovarian response during IVF than the ability to conceive naturally $[54,55]$. Early follicular FSH and $E_{2}$, generally drawn on day 3 of the menstrual cycle, require no advanced training to interpret and therefore can be obtained and interpreted by general practitioners; however, they have significant inter-cycle and intra-cycle variability and require a functional hypothalamic pituitary ovarian axis [56]. Day 3 elevated FSH is indicative of diminished ovarian reserve and is attributed to low $E_{2}$ and inhibin $B$ production from ovarian follicles thereby resulting in diminished hypothalamic suppression. Normal FSH levels in the presence of elevated $E_{2}$ may also be indicative of low ovarian reserve as this is related to premature follicle selection and premature elevation of $E_{2}$, thus negatively inhibiting the hypothalamus and masking diminished ovarian reserve.

AFC is the sum of all the ovarian follicles between 2 and $10 \mathrm{~mm}$ visualized on ultrasound. AFC count has good specificity, however, poor sensitivity for prediction of ovarian response to stimulation and pregnancy outcomes during IVF. AFC is easy to perform by a trained sonographer, however, its limitations are that it can have significant inter-observer variability and obesity can adversely affect the measurements [57].

$\mathrm{AMH}$ is secreted by the granulosa cells of the pre-antral and small antral follicles and likely plays a role in limiting follicular recruitment of follicles from the primordial pool, partially by attenuating their response to FSH [58]. AMH null mice reveal accelerated follicular depletion [59]. The benefits of AMH measurements are that they can be tested anytime throughout the cycle and are directly secreted form the ovary. Several studies have shown that AMH may also predict the timing of menopause [60-62]. Limitations of AMH, similar to day $3 \mathrm{FSH} / \mathrm{E}_{2}$ and $\mathrm{AFC}$, are that they are more reliable to predict ovarian response and IVF outcomes rather than the ability to conceive naturally, and can be falsely elevated and decreased by many reproductive, environmental, and lifestyle factors [56, 63]. One factor common to reproductive aged women is the use of oral contraception (OCP). AMH can be falsely decreased in women using OCP's and it is recommended to wait 3-4 months following discontinuation of OCP to measure AMH levels [56].

A recently published prospective time-to-pregnancy study evaluating the predictive value of $\mathrm{AMH}$ and Day $3 \mathrm{FSH}$ in over 700 women aged 30-44 y/o, without a history of infertility, and attempting to conceive $\leq 3$ months revealed that low AMH and elevated FSH were not predictive of the ability to conceive up to 12 menstrual cycles [64]. It is important to note that in the older age group (38-44 y/o), low AMH was also not predictive of time to pregnancy. A significant limitation of this study worth noting is that pregnancy was defined as a positive pregnancy test and if followed to live birth, it is possible that those in the low AMH/elevated FSH groups may have experienced pregnancy loss more often and therefore live birth rates may have differed. 


\section{Oocyte cryopreservation}

There are no known treatments to circumvent the natural decline in fecundity that occurs with ovarian aging. Using donor oocytes is an option for women unable to conceive with their own oocytes; however, this may not be acceptable to many women and is not available and/ or legal in many countries. Embryo cryopreservation and OC are available options, which may enable women to become pregnant with their own oocytes after their reproductive potential has significantly diminished. The focus of the following paragraphs will focus on $\mathrm{OC}$ as the majority of women that delay childbearing report lack of a partner as a reason for delay $[65,66]$.

The first pregnancy following OC was in 1986 [67]. Over 2 decades later, in 2012, the "experimental" label for OC was removed by the American Society for Reproductive Medicine (ASRM) and the European Society for $\mathrm{Hu}-$ man Reproduction and Embryology (ESHRE) [68, 69]. Factors that led to removing the "experimental" label included improved success rates of $\mathrm{OC}$ and reassuring safety data relating to offspring born following OC. Since the removal of the experimental label by ASRM, OC has increased $18.6 \%$ in 1 year from 6123 cycles in 2014 to 7518 cycles in 2015 [70, 71].

\section{Slow freeze versus Vitrification}

A major advancement in $\mathrm{OC}$ was a shift from the slow freeze method of cryopreservation to vitrification. Vitrification is an ultra-fast cooling method for cryopreservation using high concentrations of cryoprotectants. Vitrification solidifies the oocyte to a glass-like state and minimizes ice crystal formation, more commonly seen with the slow freeze technique. The oocyte is particularly vulnerable to damage from ice crystal formation due to its high water content and its sensitive meiotic spindle apparatus [72, 73]. A randomized controlled trial reported improved oocyte survival rates, fertilization rates, and clinical pregnancy rates with vitrification compared to slow freeze [74]. Post-thaw survival rates using vitrification have been reported to be as high as $96.9 \%$ [75]. Pregnancy rates following OC have been reported to be similar to those following fresh oocyte insemination by multiple randomized controlled trials [76-78]. Although reports of high oocyte survival rates and similar pregnancy rates with OC compared to fresh insemination have been reported, these high success rates may be attributed to publication bias and therefore the success rates may not be reproducible by all centers. Data from 30,160 donor oocyte cycles reported to the Society for Assisted Reproductive Technology from 2013 to 2015 revealed that pregnancy rates from cycles following $\mathrm{OC}$ in donor cycles were significantly lower compared to donor cycles involving freshly inseminated oocytes [79].

\section{Indications for oocyte cryopreservation}

Aside from preventive measures for age related fertility decline, women at risk for an accelerated rate of fertility decline due to medical reasons may benefit from OC. Women diagnosed with malignant diseases that will undergo chemotherapy and/or pelvic irradiation are at significant risk for premature ovarian failure and certainly warrant counseling on OC. Women with severe endometriosis/endometriomas may be at risk for an accelerated rate of follicular decline. A study has found that ovarian cortex from ovaries with endometriomas have significantly decreased follicular densities compared to contralateral ovaries without endometriomas [53]. In a similar study, cortex from ovaries with endometriomas had significantly more atretic early follicles compared to contralateral ovaries without endometriomas [80]. Additionally, a systematic review and meta-analysis revealed that excision of endometriomas could negatively impact ovarian reserve [81]. Women with a family history of mental retardation or a known family history of Fragile $\mathrm{X}$ Syndrome may benefit from Fragile X carrier testing as women with premutations (55 to 200 repeats) are at increase risk of premature ovarian failure (up to 21\%) and therefore could benefit from OC [52]. Other genetic disorders include patients with Turners or Turners mosaic and those with a strong family history of premature ovarian failure. Pedigree studies have shown a familial link to idiopathic premature ovarian failure as multiple genes likely play a role that are not fully understood [82]. Women undergoing female to male gender transition often undergo bilateral salpingoophorectomy and therefore should be offered $\mathrm{OC}$ as pregnancies have been achieved in this cohort of patients following transition [83]. Other indications for OC include inability to produce sperm at time of ooycte retrieval, ethical dilemma to producing and cryopreserving embryos, and circumventing legal implications with embryo cryopreservation in the event of divorce.

\section{Age at which to freeze oocytes}

A question frequently asked by both practitioners and patients is the ideal age at which to freeze oocytes. Although no good answer exists as every patient has unique biological and social circumstances, there is some evidence available to guide the discussion. Similar to IVF, success rates following $\mathrm{OC}$ may be related to the age at which the oocytes were frozen. A retrospective multicenter study spanning an 8-year time period evaluating 1468 women undergoing elective oocyte cryopreservation for reasons other than a diagnosis of cancer revealed that 191 (13\%) presented to thaw and inseminate their oocytes [84]. Of those that did, the oocyte survival rate in women $\leq 35 \mathrm{y} / \mathrm{o}$ and $\geq 36$ y/o were $94.6 \%$ vs. $82.4 \%$, respectively [84]. Additionally, differences in live birth rates for women 
undergoing $\mathrm{OC} \leq 35 \mathrm{y} / \mathrm{o}$ and $\geq 36 \mathrm{y} / \mathrm{o}$ were significantly different, with $50 \%$ and $22.9 \%$, respectively [84]. In this study, age was associated with success even in the cohort of women undergoing $\mathrm{OC} \leq 35 \mathrm{y} / \mathrm{o}$, with live birth rates of $100 \% \leq 29 \mathrm{y} / \mathrm{o}, 45 \%$ at $30-34 \mathrm{y} / \mathrm{o}, 28.5 \%$ at $35-$ $39 \mathrm{y} / \mathrm{o}$, and $3.7 \%$ at $\geq 40 \mathrm{y} / \mathrm{o}$ [84]. Despite a clear trend in success rates with age at which a woman undergoes $\mathrm{OC}$, the majority of women that undergo elective OC are doing so after a significant decline in fertility begins to occur, with three studies revealing average ages between $36 \mathrm{y} / \mathrm{o}$ and $38 \mathrm{y} / \mathrm{o}[65,84,85]$.

In women considering $\mathrm{OC}$, a frequent question is how many oocytes should be frozen. Although there is no precise answer to this question as every women presenting for $\mathrm{OC}$ will be at a different age and have different future childbearing desires, there is some data to guide a discussion. Clinical pregnancy rate per oocyte thaw have been shown to range between 4.5 and $12 \%$ [68]. A recent mathematical model based on live birth rates from a single institution from women with presumably normal ovarian reserve undergoing intracytoplasmic sperm injection (ICSI) due to male factor infertility or tubal factor infertility, revealed that for women 34,37 , and $42 \mathrm{y} /$ o, they would need to freeze 10, 20, and 61 mature oocytes respectively to have a $75 \%$ likelihood of having at least 1 live birth [86]. If incorporating this or another model into a OC counseling session, it is important to discuss the possibility of needing multiple controlled ovarian hyperstimulation $(\mathrm{COH})$ cycles to obtain the number of oocytes the patient desires. For example, based on the above model, a 37 y/o may feel comfortable with freezing 20 mature oocytes for a $75 \%$ chance of at least 1 live birth, however, obtaining 20 mature oocytes may require 2 or $3 \mathrm{COH}$ cycles. Additionally, it must be stressed to patients undergoing $\mathrm{OC}$ that although mathematical models exist to guide a discussion of how many oocytes to freeze in order to assure a certain percentage of success, OC is not an $100 \%$ insurance policy to achieve a genetically related offspring and that there is a possibility of failure despite "adequate" amounts of frozen oocytes.

\section{Cost considerations of $\mathrm{OC}$}

Cost of oocyte cryopreservation may vary widely from infertility center to infertility center and is an important part of a counseling session for patients desiring elective $\mathrm{OC}$ as this is frequently not covered by insurance for reasons other than a medical indication. Although OC incurs an expense, if performed early it may be more cost-effective and successful for women desiring to postpone their childbearing to their late 30's or early 40's. One model has shown that OC would be more cost-effective and successful if performed at $35 \mathrm{y} / \mathrm{o}$ in women looking to prolong their childbearing until $40 \mathrm{y} / \mathrm{o}$, whereas another model revealed that $\mathrm{OC}$ was most cost-effective and successful in achieving a live birth if performed by $37 \mathrm{y} / \mathrm{o}$ [87, 88]. In these models, the assumption is that the patient will return for 1 child at an older age, however, many patients may desire $>1$ child. It is important to discuss with patients how many children they desire, as the more children they desire, the more cost-effective it may be to undergo $\mathrm{OC}$ at a younger age. Other important cost considerations that must be discussed with patients are the annual cost of storing the oocytes, cost of thawing and inseminating oocytes via ICSI, and eventual uterine transfer of the embryos. Additionally, ethical considerations of $\mathrm{OC}$ must be extensively discussed, documented, and consented including duration of storage of oocytes and disposition of oocytes in the event of death or incapacitation of the patient.

\section{Risks of OC}

Risks of OC must be discussed with patients undergoing the procedure. Major complications for patients are low $(<1 \%)$ and include risks during oocyte retrieval including infection, damage to organs, blood loss, ovarian torsion, and risks related to anaesthesia [89-91]. A more common risk that must be discussed is ovarian hyperstimulation syndrome (OHSS), a complication specific to $\mathrm{COH}$. Although mild and moderate OHSS is burdensome for the patient, severe OHSS is potentially life-threatening and most concerning. The risk varies depending on the definition and study, but has generally been reported to be $<5 \%$ [92]. Severe OHSS is more common following $\mathrm{COH}$ with embryo transfer resulting in a pregnancy and is rare in the absence of pregnancy. Women undergoing $\mathrm{OC}$, by definition, will not undergo a fresh embryo transfer and therefore the risk of severe OHSS is significantly less than patients undergoing IVF/ ICSI with subsequent fresh embryo transfer. A study evaluating 4052 oocyte donors revealed a risk of moderate to severe OHSS of $<1 \%$, which was eliminated with the use of a $\mathrm{GnRH}$ antagonist protocol and GnRH agonist trigger [93].

A frequent concern for women undergoing $\mathrm{OC}$ is whether there is any risk to offspring born following OC. Although data are limited and more long-term data are needed, there does not appear to be any risk to offspring related to OC. A study of 1027 children born from 804 pregnancies using vitrified oocytes compared to 1224 children from 996 pregnancies with IVF using fresh oocytes revealed no significant differences in obstetrical outcomes, gestational age at delivery, birthweight, birth defects, APGAR scores, or perinatal mortality between cryopreserved and fresh oocytes [94]. Another study evaluating 936 infants born from both vitrified and slow freeze oocytes revealed an anomaly rate of $1.3 \%$, similar to the anomaly rate in the general population [95]. Additionally 
rates of embryo aneuoploidy have been reported to be similar following oocyte cryopreservation and IVF using fresh oocytes [96, 97].

\section{Education and increasing awareness towards reproductive aging and elective $O C$}

It is well documented that reproductive aged woman frequently underestimate the impact that increasing age has on the ability to conceive and overestimate the success of IVF to circumvent infertility. Sup-optimal physician counseling may be partly related to this as a study evaluating US OB/GYN residents reveals that they too have suboptimal knowledge relating to reproductive aging and IVF success rates [44]. A study by Hodes-Wertz et al. evaluating 183 women who underwent elective oocyte cryopreservation revealed that only $25 \%$ heard of the procedure from their $\mathrm{OB} / \mathrm{GYN}$, and $79 \%$ wished that they had undergone the procedure at an earlier age, with the most common reason for delay being unaware of the procedure or feeling that the technology was not readily available [65].

Although elective $\mathrm{OC}$ is generally not covered by private health insurances in the U.S. or government health policies in Europe, attitudes and policies relating to covering elective $\mathrm{OC}$ are shifting. Large companies such as Facebook and Apple are offering their employees elective oocyte cryopreservation [98]. Additionally, some countries such as Israel endorse elective $\mathrm{OC}$ for prevention of age related fertility decline and consider it "preventative medicine" [99].

Improved methods to increase awareness relating to reproductive aging and the availability of $\mathrm{OC}$ are warranted. A greater emphasis by OB/GYN's to counsel patients on age related fertility decline during annual exams and implementing these discussions as part of sexual education may help increase awareness. Additionally using media as a platform to discuss these important issues may be of importance as $42.3 \%$ of those that underwent elective $\mathrm{OC}$ in the above study by Hodes-Wertz et al. heard about the technology through media outlets [65]. Having a separate clinic to counsel women on reproductive aging may also be of benefit. A Fertility Assessment and Counseling Clinic was established in Copenhagen, Denmark, with a goal of providing pro-fertility individual assessment and guidance to women and men [100]. At the time of publication $>1200$ women and men attended the clinic and filled out a survey. Ninety-nine percent of women found the clinic helpful with approximately $70 \%$ wanting to know how long they could postpone their childbearing, and after consultation, $35 \%$ of women stating that they would advance the age at which to become pregnant.

\section{Conclusion}

The adverse effect of reproductive aging on fecundity has been well documented for centuries. With delayed motherhood becoming a norm in many countries, involuntary childlessness will likely continue to rise. This may not only be detrimental to a woman's overall well-being but may also have adverse economic consequences to future generations. Despite this, reproductive aged women are frequently unaware and underestimate the effects of reproductive aging, and/or overestimate the success rates of IVF to circumvent infertility, particularly in older aged women. Increase awareness and education at earlier ages are warranted so that women can make informed decisions relating to their reproductive potential. For the first time in history, OC provides a way of increasing a woman's reproductive autonomy for those desiring genetically related children towards the end of their reproductive lifespan. The past several decades has seen an increase in effective available methods to delay childbearing including greater access to contraception and safe pregnancy terminations, however, an integral part of a woman's reproductive autonomy is pro-fertility knowledge. Discussions relating to reproductive aging and oocyte cryopreservation will enable woman to make informed decisions relating to their reproductive potential and increase their reproductive autonomy.

\section{Abbreviations}

AFC: Antral follicle count; AMH: Anti-mullerian hormone; $\mathrm{COH}$ : Controlled ovarian hyperstimulation; $\mathrm{E}_{2}$ : Estradiol; FSH: Follicle stimulating hormone; ICSI: Intracytoplasmic sperm injection; IUI: Intrauterine Insemination; IVF: In vitro fertilization; OB/GYN: Obstetrics and Gynecology; OCP: Oral

contraception; OHSS: Ovarian hyperstimulation syndrome

\section{Authors contributions}

RF and SJ both contributed to writing and editing the manuscript. RF and SJ read and approved the final manuscript.

Ethics approval and consent to participate

Not applicable.

Consent for publication

Not applicable.

Competing interests

The authors declare that they have no competing interests.

\section{Publisher's Note}

Springer Nature remains neutral with regard to jurisdictional claims in published maps and institutional affiliations.

Received: 9 May 2018 Accepted: 2 August 2018

Published online: 11 August 2018

\footnotetext{
References

1. Prevention CfDCa. 2002. Available from: https://www.cdc.gov/nchs/data/ nvsr/nvsr51/nvsr51_01.pdf. Accessed 12/23/2017.

2. Prevention CfDCa. 2016. Available from: https://www.cdc.gov/nchs/data/ databriefs/db232.pdf. Accessed 12/23/2017.

3. Prevention CfDCa. 2014. Available from: https://www.cdc.gov/nchs/data/ databriefs/db152_table.pdf\#1. Accessed 12/23/2017.

4. mothers. SCTDSHadocooft. 2008. Available from: https://www12.statcan.gc. $\mathrm{ca} /$ census-recensement/index-eng. $\mathrm{fm}$ ?MM=1. Accessed 31 Dec 2017.

5. Eurostat. Women in the EU gave birth to their first child at almost 29 years of age on average 2015. Available from: http://ec.europa.eu/eurostat/ documents/2995521/6829228/3-13052015-CP-EN.pdf/7e9007fb-3ca9-445f96eb-fd75d6792965. Accessed 12/31/2017.

6. Group ECW. Social determinants of human reproduction. Hum Reprod. 2001;16(7):1518-26.
} 
7. Nargund G. Declining birth rate in developed countries: a radical policy rethink is required. Facts Views Vis Obgyn. 2009;1(3):191-3.

8. Ziebe S, Devroey P. State of ARTWG. Assisted reproductive technologies are an integrated part of national strategies addressing demographic and reproductive challenges. Hum Reprod Update. 2008;14(6):583-92.

9. Baker TG. A quantitative and cytological study of germ cells in human ovaries. Proc R Soc Lond B Biol Sci. 1963;158:417-33.

10. Block E. Quantitative morphological investigations of the follicular system in women; variations at different ages. Acta Anat (Basel). 1952;14(1-2):108-23.

11. Pellestor F, Andreo B, Arnal F, Humeau C, Demaille J. Maternal aging and chromosomal abnormalities: new data drawn from in vitro unfertilized human oocytes. Hum Genet. 2003;112(2):195-203.

12. Franasiak JM, Forman EJ, Hong KH, Werner MD, Upham KM, Treff NR, et al. The nature of aneuploidy with increasing age of the female partner: a review of 15,169 consecutive trophectoderm biopsies evaluated with comprehensive chromosomal screening. Fertility and sterility. 2014;101:3:656-63 e1.

13. Volarcik K, Sheean L, Goldfarb J, Woods L, Abdul-Karim FW, Hunt P. The meiotic competence of in-vitro matured human oocytes is influenced by donor age: evidence that folliculogenesis is compromised in the reproductively aged ovary. Hum Reprod. 1998;13(1):154-60,

14. Battaglia DE, Goodwin P, Klein NA, Soules MR. Influence of maternal age on meiotic spindle assembly in oocytes from naturally cycling women. Hum Reprod. 1996;11(10):2217-22.

15. Menken J, Trussell J, Larsen U. Age and infertility. Science. 1986;233(4771): 1389-94.

16. Tietze C. Reproductive span and rate of reproduction among Hutterite women. Fertil Steril. 1957;8(1):89-97.

17. American College of $\mathrm{O}$, Gynecologists Committee on Gynecologic $\mathrm{P}$, Practice C. Female age-related fertility decline. Committee Opinion No. 589. Fertility and sterility. 2014;101:3:633-4.

18. Steiner AZ, Jukic AM. Impact of female age and nulligravidity on fecundity in an older reproductive age cohort. Fertility and sterility. 2016;105:6:1584-8 e1.

19. Wesselink AK, Rothman K, Hatch EE, Mikkelsen EM, Sorensen HT, Wise LA. Age and fecundability in a North American preconception cohort study. American journal of obstetrics and gynecology. 2017;217:6:667 e1- e8.

20. Rothman KJ, Wise LA, Sorensen HT, Riis AH, Mikkelsen EM, Hatch EE. Volitional determinants and age-related decline in fecundability: a general population prospective cohort study in Denmark. Fertil Steril. 2013;99(7): 1958-64.

21. Schwartz D, Mayaux MJ. Female fecundity as a function of age: results of artificial insemination in 2193 nulliparous women with azoospermic husbands. Federation CECOS. N Engl J Med. 1982;306(7):404-6.

22. van Noord-Zaadstra BM, Looman CW, Alsbach H, Habbema JD, te Velde ER, Karbaat J. Delaying childbearing: effect of age on fecundity and outcome of pregnancy. BMJ. 1991;302(6789):1361-5.

23. Wyndham N, Marin Figueira PG, Patrizio P. A persistent misperception: assisted reproductive technology can reverse the "aged biological clock". Fertil Steril. 2012;97(5):1044-7.

24. Prevention CfDCa. 2017. Available from: https://www.cdc.gov/art/reports/ 2015/national-summary.html. Accessed 12/23/2017.

25. Cil AP, Turkgeldi L, Seli E. Oocyte cryopreservation as a preventive measure for age-related fertility loss. Semin Reprod Med. 2015;33(6):429-35.

26. Jacobsson B, Ladfors L, Milsom I. Advanced maternal age and adverse perinatal outcome. Obstet Gynecol. 2004;104(4):727-33.

27. Cleary-Goldman J, Malone FD, Vidaver J, Ball RH, Nyberg DA, Comstock CH, et al. Impact of maternal age on obstetric outcome. Obstet Gynecol. 2005; 105(5 Pt 1):983-90.

28. Laopaiboon M, Lumbiganon P, Intarut N, Mori R, Ganchimeg T, Vogel JP, et al. Advanced maternal age and pregnancy outcomes: a multicountry assessment. BJOG : an international journal of obstetrics and gynaecology. 2014;121(Suppl 1):49-56.

29. Gilbert WM, Nesbitt TS, Danielsen B. Childbearing beyond age 40: pregnancy outcome in 24,032 cases. Obstet Gynecol. 1999;93(1):9-14.

30. Hoffman MC, Jeffers S, Carter J, Duthely L, Cotter A, Gonzalez-Quintero $\mathrm{VH}$. Pregnancy at or beyond age 40 years is associated with an increased risk of fetal death and other adverse outcomes. Am J Obstet Gynecol. 2007;196(5):e11-3.

31. Berkowitz GS, Skovron ML, Lapinski RH, Berkowitz RL. Delayed childbearing and the outcome of pregnancy. N Engl J Med. 1990;322(10):659-64.
32. Lean SC, Derricott H, Jones RL, Heazell AEP. Advanced maternal age and adverse pregnancy outcomes: A systematic review and meta-analysis. Plos one. 2017;12:10:e0186287.

33. Nybo Andersen AM, Wohlfahrt J, Christens P, Olsen J, Melbye M. Maternal age and fetal loss: population based register linkage study. BMJ. 2000; 320(7251):1708-12.

34. Bardos J, Hercz D, Friedenthal J, Missmer SA, Williams Z. A national survey on public perceptions of miscarriage. Obstet Gynecol. 2015;125(6):1313-20.

35. Chan CH, Chan TH, Peterson BD, Lampic C, Tam MY. Intentions and attitudes towards parenthood and fertility awareness among Chinese university students in Hong Kong: a comparison with western samples. Hum Reprod. 2015;30(2):364-72.

36. Peterson BD, Pirritano M, Tucker L, Lampic C. Fertility awareness and parenting attitudes among American male and female undergraduate university students. Hum Reprod. 2012;27(5):1375-82.

37. Lundsberg LS, Pal L, Gariepy AM, Xu X, Chu MC, Illuzzi JL. Knowledge, attitudes, and practices regarding conception and fertility: a populationbased survey among reproductive-age United States women. Fertil Steril. 2014:101(3):767-74.

38. Bretherick KL, Fairbrother N, Avila L, Harbord SH, Robinson WP. Fertility and aging: do reproductive-aged Canadian women know what they need to know? Fertil Steril. 2010;93(7):2162-8.

39. Hashiloni-Dolev Y, Kaplan A, Shkedi-Rafid S. The fertility myth: Israeli students' knowledge regarding age-related fertility decline and late pregnancies in an era of assisted reproduction technology. Hum Reprod. 2011;26(11):3045-53.

40. Tough S, Benzies K, Newburn-Cook C, Tofflemire K, Fraser-Lee N, Faber A, et al. What do women know about the risks of delayed childbearing? Can J Public Health. 2006;97(4):330-4.

41. Adashi EY, Cohen J, Hamberger L, Jones HW Jr, de Kretser DM, Lunenfeld $B$, et al. Public perception on infertility and its treatment: an international survey. The Bertarelli Foundation scientific board. Hum Reprod. 2000;15(2):330-4.

42. Lampic C, Svanberg AS, Karlstrom P, Tyden T. Fertility awareness, intentions concerning childbearing, and attitudes towards parenthood among female and male academics. Hum Reprod. 2006;21(2):558-64.

43. Bunting L, Tsibulsky I, Boivin J. Fertility knowledge and beliefs about fertility treatment: findings from the international fertility decision-making study. Hum Reprod. 2013;28(2):385-97.

44. Yu L, Peterson B, Inhorn MC, Boehm JK, Patrizio P. Knowledge, attitudes, and intentions toward fertility awareness and oocyte cryopreservation among obstetrics and gynecology resident physicians. Hum Reprod. 2016; 31(2):403-11.

45. Grady D. Clinical practice. Management of menopausal symptoms. N Engl J Med. 2006:355(22):2338-47.

46. Nikolaou D, Templeton A. Early ovarian ageing: a hypothesis. Detection and clinical relevance. Hum Reprod. 2003:18(6):1137-9.

47. Coulam CB, Adamson SC, Annegers JF. Incidence of premature ovarian failure. Obstet Gynecol. 1986;67(4):604-6.

48. Barnhart K, Dunsmoor-Su R, Coutifaris C. Effect of endometriosis on in vitro fertilization. Fertil Steril. 2002;77(6):1148-55.

49. Augood C, Duckitt K, Templeton AA. Smoking and female infertility: a systematic review and meta-analysis. Hum Reprod. 1998;13(6):1532-9.

50. Cramer DW, Xu H, Harlow BL. Family history as a predictor of early menopause. Fertil Steril. 1995;64(4):740-5.

51. Schattman GL. Cryopreservation of Oocytes. Author replies. The New England journal of medicine. 2016:374:3:288.

52. Sherman SL. Premature ovarian failure in the fragile X syndrome. Am J Med Genet. 2000;97(3):189-94.

53. Kitajima M, Defrere S, Dolmans MM, Colette S, Squifflet J, Van Langendonckt $\mathrm{A}$, et al. Endometriomas as a possible cause of reduced ovarian reserve in women with endometriosis. Fertil Steril. 2011;96(3):685-91.

54. Muasher SJ, Oehninger S, Simonetti S, Matta J, Ellis LM, Liu HC, et al. The value of basal and/or stimulated serum gonadotropin levels in prediction of stimulation response and in vitro fertilization outcome. Fertil Steril. 1988; 50(2):298-307.

55. Scott RT, Toner JP, Muasher SJ, Oehninger S, Robinson S, Rosenwaks Z. Follicle-stimulating hormone levels on cycle day 3 are predictive of in vitro fertilization outcome. Fertil Steril. 1989;51(4):651-4.

56. Tal R, Seifer DB. Ovarian reserve testing: A user's guide. American journal of obstetrics and gynecology. 2017;https://doi.org/10.1016/j.ajog.2017.02.027. 
57. Broekmans FJ, Kwee J, Hendriks DJ, Mol BW, Lambalk CB. A systematic review of tests predicting ovarian reserve and IVF outcome. Hum Reprod Update. 2006;12(6):685-718.

58. Durlinger AL, Gruijters MJ, Kramer P, Karels B, Kumar TR, Matzuk MM, et al. Anti-Mullerian hormone attenuates the effects of FSH on follicle development in the mouse ovary. Endocrinology. 2001;142(11):4891-9.

59. Durlinger AL, Kramer P, Karels B, de Jong FH, Uilenbroek JT, Grootegoed JA, et al. Control of primordial follicle recruitment by anti-Mullerian hormone in the mouse ovary. Endocrinology. 1999;140(12):5789-96.

60. Broer SL, Eijkemans MJ, Scheffer GJ, van Rooij IA, de Vet A, Themmen AP, et al. Anti-mullerian hormone predicts menopause: a long-term follow-up study in normoovulatory women. J Clin Endocrinol Metab. 2011;96(8):2532-9.

61. Freeman EW, Sammel MD, Lin H, Boorman DW, Gracia CR. Contribution of the rate of change of antimullerian hormone in estimating time to menopause for late reproductive-age women. Fertility and sterility. 2012;98: 5:1254-9 e1-2.

62. Freeman EW, Sammel MD, Lin H, Gracia CR. Anti-mullerian hormone as a predictor of time to menopause in late reproductive age women. J Clin Endocrinol Metab. 2012;97(5):1673-80.

63. La Marca A, Sighinolfi G, Radi D, Argento C, Baraldi E, Artenisio AC, et al. Anti-Mullerian hormone (AMH) as a predictive marker in assisted reproductive technology (ART). Hum Reprod Update. 2010;16(2):113-30.

64. Steiner AZ, Pritchard D, Stanczyk FZ, Kesner JS, Meadows JW, Herring AH, et al. Association between biomarkers of ovarian reserve and infertility among older women of reproductive age. JAMA. 2017;318(14):1367-76.

65. Hodes-Wertz B, Druckenmiller S, Smith M, Noyes N. What do reproductiveage women who undergo oocyte cryopreservation think about the process as a means to preserve fertility? Fertil Steril. 2013;100(5):1343-9.

66. Hammarberg K, Clarke VE. Reasons for delaying childbearing--a survey of women aged over 35 years seeking assisted reproductive technology. Aust Fam Physician. 2005;34:3:187-8, 206.

67. Chen C. Pregnancy after human oocyte cryopreservation. Lancet. 1986; 1(8486):884-6

68. Practice Committees of American Society for Reproductive M, Society for Assisted Reproductive T. Mature oocyte cryopreservation: a guideline. Fertility and sterility. 2013;99:1:37-43.

69. Ethics ETFo, Law, Dondorp W, de Wert G, Pennings G, Shenfield F, et al. Oocyte cryopreservation for age-related fertility loss. Human reproduction. 2012;27:5:1231-7.

70. Technologies SfAR. 2017. Available from: https://www.sartcorsonline.com/ rptCSR_PublicMultYear.aspx?reporting Year=2015. Accessed 1/29/2018.

71. Technologies SfAR. 2017. Available from: https://www.sartcorsonline.com/ rptCSR_PublicMultYear.aspx?reportingYear=2014. Accessed 1/229/2018.

72. Bromfield JJ, Coticchio G, Hutt K, Sciajno R, Borini A, Albertini DF. Meiotic spindle dynamics in human oocytes following slow-cooling cryopreservation. Hum Reprod. 2009;24(9):2114-23.

73. Shaw JM, Oranratnachai A, Trounson AO. Fundamental cryobiology of mammalian oocytes and ovarian tissue. Theriogenology. 2000;53(1):59-72.

74. Smith GD, Serafini PC, Fioravanti J, Yadid I, Coslovsky M, Hassun P, et al. Prospective randomized comparison of human oocyte cryopreservation with slow-rate freezing or vitrification. Fertil Steril. 2010;94(6):2088-95.

75. Cobo A, Kuwayama M, Perez S, Ruiz A, Pellicer A, Remohi J. Comparison of concomitant outcome achieved with fresh and cryopreserved donor oocytes vitrified by the Cryotop method. Fertil Steril. 2008;89(6):1657-64.

76. Cobo A, Meseguer M, Remohi J, Pellicer A. Use of cryo-banked oocytes in an ovum donation programme: a prospective, randomized, controlled, clinical trial. Hum Reprod. 2010;25(9):2239-46.

77. Parmegiani L, Cognigni GE, Bernardi S, Cuomo S, Ciampaglia W, Infante FE, et al. Efficiency of aseptic open vitrification and hermetical cryostorage of human oocytes. Reprod BioMed Online. 2011;23(4):505-12.

78. Rienzi L, Romano S, Albricci L, Maggiulli R, Capalbo A, Baroni E, et al. Embryo development of fresh 'versus' vitrified metaphase II oocytes after ICSI: a prospective randomized sibling-oocyte study. Hum Reprod. 2010; 25(1):66-73

79. Kushnir VA, Darmon SK, Barad DH, Gleicher N. New national outcome data on fresh versus cryopreserved donor oocytes. J Ovarian Res. 2018;11:1:2

80. Kitajima M, Dolmans MM, Donnez O, Masuzaki H, Soares M, Donnez J. Enhanced follicular recruitment and atresia in cortex derived from ovaries with endometriomas. Fertil Steril. 2014;101(4):1031-7.
81. Raffi F, Metwally M, Amer S. The impact of excision of ovarian endometrioma on ovarian reserve: a systematic review and meta-analysis. J Clin Endocrinol Metab. 2012;97(9):3146-54.

82. van Kasteren $Y M$, Hundscheid RD, Smits AP, Cremers FP, van Zonneveld $P$, Braat DD. Familial idiopathic premature ovarian failure: an overrated and underestimated genetic disease? Hum Reprod. 1999;14(10):2455-9.

83. Maxwell S, Noyes N, Keefe D, Berkeley AS, Goldman KN. Pregnancy outcomes after fertility preservation in transgender men. Obstet Gynecol. 2017;129(6):1031-4.

84. Cobo A, Garcia-Velasco JA, Coello A, Domingo J, Pellicer A, Remohi J. Oocyte vitrification as an efficient option for elective fertility preservation. Fertility and sterility. 2016;105:3:755-64 e8.

85. Baldwin K, Culley L, Hudson N, Mitchell H, Lavery S. Oocyte cryopreservation for social reasons: demographic profile and disposal intentions of UK users. Reprod BioMed Online. 2015:31(2):239-45.

86. Goldman RH, Racowsky C, Farland LV, Munne S, Ribustello L, Fox JH. Predicting the likelihood of live birth for elective oocyte cryopreservation: a counseling tool for physicians and patients. Human reproduction. 2017; https://doi.org/10.1093/humrep/dex008:1-7.

87. van Loendersloot LL, Moolenaar LM, Mol BW, Repping S, van der Veen F, Goddijn M. Expanding reproductive lifespan: a cost-effectiveness study on oocyte freezing. Hum Reprod. 2011;26(11):3054-60.

88. Mesen TB, Mersereau JE, Kane JB, Steiner AZ. Optimal timing for elective egg freezing. Fertility and sterility. 2015;103:6:1551-6 e1-4.

89. Maxwell KN, Cholst IN, Rosenwaks Z. The incidence of both serious and minor complications in young women undergoing oocyte donation. Fertil Steril. 2008;90(6):2165-71.

90. Aragona C, Mohamed MA, Espinola MS, Linari A, Pecorini F, Micara G, et al. Clinical complications after transvaginal oocyte retrieval in 7,098 IVF cycles. Fertil Steril. 2011;95(1):293-4.

91. Roest J, Mous HV, Zeilmaker GH, Verhoeff A. The incidence of major clinical complications in a Dutch transport IVF programme. Hum Reprod Update. 1996:2(4):345-53.

92. Practice Committee of the American Society for Reproductive Medicine. Electronic address Aao, Practice Committee of the American Society for Reproductive M. Prevention and treatment of moderate and severe ovarian hyperstimulation syndrome: a guideline. Fertility and sterility. 2016;106:7:1634-47.

93. Bodri D, Guillen JJ, Polo A, Trullenque M, Esteve C, Coll O. Complications related to ovarian stimulation and oocyte retrieval in 4052 oocyte donor cycles. Reprod BioMed Online. 2008;17(2):237-43.

94. Cobo A, Serra V, Garrido N, Olmo I, Pellicer A, Remohi J. Obstetric and perinatal outcome of babies born from vitrified oocytes. Fertility and sterility. 2014;102:4:1006-15 e4.

95. Noyes N, Porcu E, Borini A. Over 900 oocyte cryopreservation babies born with no apparent increase in congenital anomalies. Reprod BioMed Online. 2009;18(6):769-76.

96. Cobo A, Rubio C, Gerli S, Ruiz A, Pellicer A, Remohi J. Use of fluorescence in situ hybridization to assess the chromosomal status of embryos obtained from cryopreserved oocytes. Fertil Steril. 2001;75(2):354-60.

97. Goldman KN, Kramer Y, Hodes-Wertz B, Noyes N, McCaffrey C, Grifo JA. Long-term cryopreservation of human oocytes does not increase embryonic aneuploidy. Fertil Steril. 2015;103(3):662-8.

98. Zoll M, Mertes H, Gupta J. Corporate giants provide fertility benefits: have they got it wrong? Eur J Obstet Gynecol Reprod Biol. 2015;195:A1-2.

99. Shkedi-Rafid S, Hashiloni-Dolev Y. Egg freezing for age-related fertility decline: preventive medicine or a further medicalization of reproduction? Analyzing the new Israeli policy. Fertil Steril. 2011;96(2):291-4

100. Hvidman HW, Petersen KB, Larsen EC, Macklon KT, Pinborg A, Nyboe AA Individual fertility assessment and pro-fertility counselling; should this be offered to women and men of reproductive age? Hum Reprod. 2015:30(1):9-15. 